\title{
SUR LE MOUVEMENT D'ENSEMBLE, AUTOUR DE SON CENTRE DE GRAVITÉ, D'UNE MASSE FLUIDE SOUMISE À L'ATTRACTION NEWTONNIENNE.
}

\author{
PAR \\ PAUL APPELL \\ à PARIS.
}

I. L'hypothèse que les planètes ont d'abord été fluides est aujourd'hui universellement admise. C'est ce qui donne une grande importance cosmogonique aux travaux de tant de géomètres sur les figures d'équilibre relatif d'une masse fluide, soumise à l'attraction de ses particules, tournant autour d'un axe de direction fixe issu du centre de gravité.

En particulier, dans ces dernières années, H. Poincarḱ et Liapounoff ont étudié les figures d'équilibre relatif d'une masse liquide, de densité $\varrho$ constante, qui tourne autour d'un axe de direction fixe passant par le centre de gravité, en supposant que les seules forces extérieures se réduisent à une pression constante s'exerçant sur la surface libre. L'objet du présent travail est de montrer que ce monvement est le seul possible, même dans le cas plus général où la masse liquide est soumise, en outre, à des attractions Newtonniennes extérieures, en supposant non seulement que $\varrho$ est constant mais aussi que la densité $\varrho$ est fonction de la seule pression $P$. En prenant le cas de $H$. Poincaré et Liapounoff j'ai déjà établi ce résultat dans le numéro i6 du quatrième volume du Traité de mécanique rationnelle que j'ai publié chez Gauthier-Villars.

2. Plaçons nous done dans le cas précis suivant: une masse fluide dans laquelle la densité $\varrho$ est fonction de la pression $P$ se meut tout d'une pièce, de telle façon que ses particules restent à des distances invariables les unes des autres: cette masse est soumise à l'attraction Newtonienne de ses particules et à 
l'attraction Newtonienne d'autres corps extérieurs qui peuvent d'ailleurs être en mouvement; sur la surface libre s'exerce une pression constante dans l'espace mais pouvant varier avee le temps $t$; la densité $\varrho$ peut varier au contraire dans l'espace intérieur, mais elle reste constante en chaque point du fluide quand $t$ varie. Nous étudions le mouvement de cette masse autour de son centre de gravité $G$, c'est à dire par rapport à des axes $G x_{1}, G y_{1}, G z_{1}$, de directions fixes issus de $G$. Appelons $G x, G y, G z$ les axes principaux d'inertie de la masse fluide par rapport à $G$; ces axes sont entraînés dans le mouvement autour de $G$. Nous emploierons la notation de Lagrange en désignant par des accents les dérivées par rapport à $t$; dans les équations nous n'écrirons pas la lettre $t$. Si on appelle $p, q, r$ les composantes de la rotation instantanée des axes $G x y z$ suivant ces axes, ces composantes pourront être des fonctions de $t$. Désignons par $V$ le potentiel de la masse mobile au point $x, y, z ; U$ le potentiel des masses extérieures au même point; $U$ peut dépendre de $t$, mais $V$ est indépendant de $t$. La masse est, à chaque instant $t$, en équilibre relatif par rapport aux axes $G x y z$; pour étudier cet équilibre relatif, appelons $a, b, c$ les composantes de la vitesse absolue du centre de gravité $G$ suivant les axes $G x y z, a, b, c$ étant certaines fonctions de $t$; la vitesse absolue de la particule fluide placée au point $x, y, z$, immobile par rapport aux axes $G x y z$, a pour composantes suivant ces mêmes axes

$$
\begin{aligned}
& u=a+q z-r y, \\
& v=b+r x-p z, \\
& w=c+p y-q x
\end{aligned}
$$

son accélération absolue $J$ a pour projections

$$
\begin{aligned}
& J_{x}=u^{\prime}+q w-r v+a, \\
& J_{y}=v^{\prime}+v u-p w+b, \\
& J_{z}=w^{\prime}+p v-q u+c,
\end{aligned}
$$

c'est à dire

$$
J_{x}=a^{\prime}+a+q c-r b+p(p x+q y+r z)-\left(p^{2}+q^{2}+r^{2}\right) x+q^{\prime} z-r^{\prime} y
$$

Il faut alors, pour l'équilibre relatif, ajouter, aux forces réelles, la force centrifuge ayant pour projections rapportées à l'unité de masse

$$
-J_{x},-J_{y},-J_{z}
$$


Appelant $f$ la constante de l'attraction universelle on a, pour l'équilibre relatif de la masse fluide, les trois équations

$$
\frac{\mathrm{I}}{\varrho} \frac{\partial P}{\partial x}=X, \quad \frac{\mathrm{I}}{\varrho} \frac{\partial P}{\partial y}=Y, \quad \frac{\mathrm{I}}{\varrho} \frac{\partial P}{\partial z}=Z
$$

où

$$
X=f \frac{\partial V}{\partial x}+f \frac{\partial U}{\partial x}-\left(a^{\prime}+a+q c-r b\right)-p(p x+q y+r z)+\left(p^{2}+q^{2}+r^{2}\right) x+r^{\prime} y-q^{\prime} z
$$

c'est à dire; si l'on pose

(2) $W=f V+f U-\left(a^{\prime}+a+q c-r b\right) x-\left(b^{\prime}+b+r a-p c\right) y-\left(c^{\prime}+c+p b-q a\right) z$

$$
\begin{aligned}
& \quad-\frac{\mathrm{I}}{2}(p x+q y+r z)^{2}+\frac{\mathrm{I}}{2}\left(p^{2}+q^{2}+r^{2}\right)\left(x^{2}+y^{2}+z^{2}\right) \\
& \left\{\begin{array}{l}
X=\frac{\partial W}{\partial x}+r^{\prime} y-q^{\prime} z \\
Y=\frac{\partial W}{\partial y}+p^{\prime} z-r^{\prime} x \\
Z=\frac{\partial W}{\partial z}+q^{\prime} x-p^{\prime} y
\end{array}\right.
\end{aligned}
$$

Si $\varrho$ est constant ou fonction de $P, X d x+Y d y+Z d z$ est la différentielle totale de $\int \frac{d P}{\varrho}$; on a done

$$
\frac{\partial Y}{\partial z}-\frac{\partial Z}{\partial y}=\mathrm{o}, \frac{\partial Z}{\partial x}-\frac{\partial X}{\partial y}=0, \frac{\partial X}{\partial y}-\frac{\partial Y}{\partial x}=0
$$

c'est à dire

$$
p^{\prime}=\mathrm{o}, q^{\prime}=\mathrm{o}, r^{\prime}=\mathrm{o}
$$

Alors $p, q, r$ sont constants et le mouvement est une rotation autour d'un axe de direction fixe. C'est ce que nous voulions établir.

On peut remarquer d'après (2) que, à l'intérieur du fluide,

$$
\Delta W=f \Delta V+f \Delta U+2\left(p^{2}+q^{2}+r^{2}\right),
$$

3-25280. Acta mathematica. 47. Imprimé le 12 août 1925. 
où $\Delta W=\frac{\partial^{2} W}{\partial x^{2}}+\frac{\partial^{2} W}{\partial y^{2}}+\frac{\partial^{2} W}{\partial z^{2}} ;$ d'autre part $\Lambda U=0, \Delta V=-4 \pi \varrho$, donc

$$
\Delta W=-4 \pi \varrho f+2\left(p^{2}+q^{2}+r^{2}\right)
$$

à l'intérieur de la masse. Si $\varrho$ est constant

$$
\begin{aligned}
\frac{\mathrm{I}}{\varrho} \Delta P & =\Delta W, \\
\Delta P & =-4 \pi \varrho^{2} f+2 \varrho\left(p^{2}+q^{2}+r^{2}\right)
\end{aligned}
$$

à l'intérieur; de plus $P$ est constant sur la surface libre et nul à l'extérieur.

3. Les forces extérieures sont telles que leur travail élémentaire $\mathfrak{T}_{\varepsilon}$ est nul pour le déplacement réel autour de $G$ de l'instant $t$ à l'instant $t+d t$. En effet la demi force vive dans le mouvement autour de $G$ étant

$$
T=\frac{1}{2}\left(A p^{2}+B q^{2}+C r^{2}\right)
$$

où $A, B, C$ sont les moments principaux d'inertie, est constante. Comme le théorème des forces vives s'applique au mouvement autour de $G$, on a

$$
d T=\mathfrak{T}_{\varepsilon}
$$

comme $T$ est constant, $\mathfrak{T}_{\varepsilon}=0$.

4. On peut, comme nous l'avons indiqué dans les Comptes rendus de l'Académie des Sciences pour la séance du 2 I juillet 1924, supposer que $\varrho$ est une fonction quelconque de $x, y, z$. Alors la forme $X d x+Y d y+Z d z$ admet un facteur intégrant et on a

$$
X\left(\frac{\partial Y}{\partial z}-\frac{\partial Z}{\partial y}\right)+Y\left(\frac{\partial Z}{\partial x}-\frac{\partial X}{\partial z}\right)+Z\left(\frac{\partial X}{\partial y}-\frac{\partial Y}{\partial x}\right)=0
$$

c'est à dire

$$
p^{\prime} X+q^{\prime} Y+r^{\prime} Z=0
$$

ou encore d'après (3)

$$
p^{\prime} \frac{\partial W}{\partial x}+q^{\prime} \frac{\partial W}{\partial y}+r^{\prime} \frac{\partial W}{\partial z}=0
$$


relation qui doit avoir lieu quels que soient $x, y, z$. Comme, dans le cas général, it ne peut exister aucune relation linéaire et homogène à coefficients indépendants de $x, y, z$, entre $\frac{\partial W}{\partial x}, \frac{\partial W}{\partial y}, \frac{\partial W}{\partial z}$, on $\mathbf{a}$, en supposant qu'une telle relation n'existe pas,

$$
p^{\prime}=\mathrm{o}, q^{\prime}=\mathrm{o}, r^{\prime}=\mathrm{o}
$$

et la conclusion est la même.

5. Il existe alors un cas exceptionnel qui doit être traité à part; c'est le cas où il y a des quantités $\alpha, \beta, \gamma$, non nulles toutes trois, ne dépendant pas de $x, y, z$ et telles que

$$
\alpha \frac{\partial W}{\partial x}+\beta \frac{\partial W}{\partial y}+\gamma \frac{\partial W}{\partial z}=0
$$

avec

$$
p^{\prime}=k \alpha, q^{\prime}=k \beta, r^{\prime}=k \gamma, k \neq 0
$$

Les équations

$$
\frac{\partial(\varrho Y)}{\partial z}-\frac{\partial(\varrho Z)}{\partial y}=0, \ldots
$$

donnent alors

$$
\left\{\begin{array}{l}
2 \varrho p^{\prime}+Y \frac{\partial \varrho}{\partial z}-Z \frac{\partial \varrho}{\partial y}=0 \\
2 \varrho q^{\prime}+Z \frac{\partial \varrho}{\partial x}-X \frac{\partial \varrho}{\partial z}=0 \\
2 \varrho r^{\prime}+X \frac{\partial \varrho}{\partial y}-Y \frac{\partial \varrho}{\partial x}=0
\end{array}\right.
$$

d'où l'on tire, en multipliant par $X, Y, Z$ et ajoutant la relation déjà obtenue

$$
p^{\prime} X+q^{\prime} Y+r^{\prime} Z=0
$$

qui conduit à (6). En remplaçant $X, Y, Z$ par leurs valeurs $\frac{\mathrm{I}}{\varrho} \frac{\partial P}{\partial x}, \frac{\mathrm{I}}{\varrho} \frac{\partial P}{\partial y}, \frac{\mathrm{I}}{\varrho} \frac{\partial P}{\partial z}$, et $p^{\prime}, q^{\prime}, r^{\prime}$ par $k \alpha, k \beta, k \gamma$ on a

$$
\alpha \frac{\partial P}{\partial x}+\beta \frac{\partial P}{\partial y}+\gamma \frac{\partial P}{\partial z}=\mathrm{o}
$$


Enfin, en multipliant les équations (8) respectivement par $\frac{\partial \varrho}{\partial x}, \frac{\partial \varrho}{\partial y}, \frac{\partial \varrho}{\partial z}$ et ajoutant, on a de même,

$$
\alpha \frac{\partial \varrho}{\partial x}+\beta \frac{\partial \varrho}{\partial y}+\gamma \frac{\partial \varrho}{\partial z}=0
$$

Comme $\alpha, \beta, \gamma$ sont supposés non nuls, le déterminant des coefficients de $\alpha, \beta, \gamma$ dans (6), (IO) et (I I) est nul:

$$
\left|\begin{array}{lll}
\frac{\partial W}{\partial x} & \frac{\partial W}{\partial y} & \frac{\partial W}{\partial z} \\
\frac{\partial P}{\partial x} & \frac{\partial P}{\partial y} & \frac{\partial P}{\partial z} \\
\frac{\partial \varrho}{\partial x} & \frac{\partial \varrho}{\partial y} & \frac{\partial \varrho}{\partial z}
\end{array}\right|=0
$$

le déterminant fonctionnel de $W, P, \varrho$ est donc nul et ces quantités sont liées par une relation

$$
F(W, P, \varrho)=\text { o, }
$$

relation qui montre qu'alors la densité $\varrho$ est fonction de $W$ et $P$. On peut également obtenir cette relation (13) par une autre voie que nous allons exposer. L'équation aux dérivées partielles (6) en $W$ donne

$$
W=\Phi(\lambda, \mu),
$$

$\Phi$ étant une fonction des deux variables $\lambda$ et $\mu$ définies par les relations

$$
\begin{gathered}
\lambda=\sqrt{\frac{k}{\alpha}}(\alpha y-\beta x), \mu=\sqrt{\frac{k}{\alpha}}(\alpha z-\gamma x), \\
\beta \mu-\gamma \lambda=\sqrt{\alpha k}(\beta z-\gamma y),
\end{gathered}
$$

$\alpha$ étant, par exemple, supposé $\neq$ o. On trouve de même

$$
\left\{\begin{array}{l}
P=\Psi(\lambda, \mu) \\
\varrho=\Pi(\lambda, \mu)
\end{array}\right.
$$

L'élimination de $\lambda$ et $\mu$ donne la relation cherchée (I3) entre $W, P$ et $\varrho$. On peut d'ailleurs écrire l'équation d'équilibre du fluide à l'instant $t$ ( $t$ constant) 


$$
\frac{\mathrm{I}}{\varrho} d P=d W+\left(r^{\prime} y-q^{\prime} z\right) d x+\left(p^{\prime} z-r^{\prime} x\right) d y+\left(q^{\prime} x-p^{\prime} y\right) d z
$$

en $y$ introduisant les variables $\lambda$ et $\mu$, après avoir remplacé $p^{\prime}, q^{\prime}, r^{\prime}$ par $k \alpha, k \beta, k \gamma$ :

$$
\frac{\mathrm{I}}{\varrho} d P=d W+\mu d \lambda-\lambda d \mu
$$

ce qui donne les deux équations

$$
\left\{\begin{array}{l}
\frac{\mathrm{I}}{\varrho} \frac{\partial P}{\partial \lambda}=\frac{\partial W}{\partial \lambda}+\mu, \\
\frac{\mathrm{I}}{\varrho} \frac{\partial P}{\partial \mu}=\frac{\partial W}{\partial \mu}-\lambda,
\end{array}\right.
$$

où $\varrho$ est une certaine fonction de $\lambda$ et $\mu$.

Dans ce cas, l'équation

$$
\varrho=\Pi(\lambda, \mu)
$$

montre que, à l'instant $t$, $\varrho$ a la même valeur en tous les points de la masse où $\lambda$ et $\mu$ ont les mêmes valeurs, c'est à dire en tous les points situés sur la droite

$$
x=\frac{p^{\prime}}{r^{\prime}} z+C, y=\frac{q^{\prime}}{r^{\prime}} z+D
$$

parallèle à la direction $\frac{x}{p^{\prime}}=\frac{y}{q^{\prime}}=\frac{\tilde{z}}{r^{\prime}}$ qui correspond à des valeurs nulles des constantes $C$ et $D$

$$
C=D=\mathrm{o}, \lambda=\mu=\mathrm{o} .
$$

La densité $\varrho$ restant la même avec le temps en des points fixes par rapport aux axes mobiles $G x y z$, la droite $\frac{x}{p^{\prime}}=\frac{y}{q^{\prime}}=\frac{z}{r^{\prime}}$ est fixe par rapport à ces axes quand $t$ varie. Done $\frac{p^{\prime}}{r^{\prime}}$ et $\frac{q^{\prime}}{r^{\prime}}$ c'est à dire $\frac{d p}{d r}$ et $\frac{d q}{d r}$ sont indépendants de $t$. On peut admettre que $\alpha, \beta, \gamma$ sont non seulement indépendants de $x, y, z$ mais indépendants de $t$, et que $k$ seul dépend de $t$. On a alors

$$
\begin{aligned}
& p=\frac{\alpha}{\gamma} r+C_{1} \\
& q=\frac{\beta}{\gamma} r+D_{3}
\end{aligned}
$$


l'extrémité de l'axe instantané est fixe ou décrit, par rapport aux axes mobiles, une droite parallèle à

$$
\frac{x}{\alpha}=\frac{y}{\beta}=\frac{z}{\gamma}
$$

Mais il semble que, dans la réalité physique, ce cas où $\alpha, \beta, \gamma$ ne sont pas nuls tous trois ne se présente pas. Car alors les surfaces

$$
W=C^{\text {te }}
$$

seraient, à chaque instant, des cylindres parallèles à la direction fixe par rapport au fluide

$$
\frac{x}{\alpha}=\frac{y}{\beta}=\frac{z}{\gamma}
$$

quelle que soit la position des corps extérieurs. Si donc on considère ce fait comme impossible on a

$$
\alpha=\beta=\gamma=0 ;
$$

$p^{\prime}, q^{\prime}, r^{\prime}$ sont alors nuls tous trois, $p, q, r$ sont constants et le fluide tourne autour d'un axe de direction fixe issu de $G$.

6. Prenons alors le cas où $p^{\prime}, q^{\prime}, r^{\prime}$ sont nuls. On peut expliquer pourquoi le soleil et certaines planètes qui sont à l'état fluide ne tournent pas tout d'une pièce: c'est ce que j'ai indiqué dans une Note présentée à l'Académie le 27 octobre 1924. Prenons l'axe de rotation, dans le mouvement autour de $G$, comme axe $G z$, les axes $G x$ et $G y$ étant liés au fluidé qui tourne d'un mouvement d'ensemble. Alors

$$
p=q=0, r=r_{0}
$$

Si on appelle $G \sigma$ le moment résultant des quantités de mouvement par rapport à $G$, les équations du mouvement sont

$$
\left\{\begin{array}{l}
\frac{d \sigma_{x}}{d t}+q \sigma_{z}-r \sigma_{y}=L, \\
\frac{d \sigma_{y}}{d t}+r \sigma_{x}-p \sigma_{z}=M, \\
\frac{d \sigma_{z}}{d t}+p \sigma_{y}-q \sigma_{x}=N
\end{array}\right.
$$


où $L, M, N$ sont les moments des forces extérieures par rapport aux axes $G x$, $G y, G z$. Soient $A, B, C, D, E, F$ les moments d'inertie et les produits d'inertie par rapport à ces axes

$$
\begin{aligned}
& A=\Sigma m\left(y^{2}+z^{2}\right), \quad B=\Sigma m\left(z^{2}+x^{2}\right), C=\Sigma m\left(x^{2}+y^{2}\right) \\
& D=\Sigma m y z, \quad E=\Sigma m z x, \quad F=\Sigma m x y \text {, }
\end{aligned}
$$

on a

$$
\begin{aligned}
& \sigma_{x}=A p-F q-E r, \\
& \sigma_{y}=B q-D r-F q, \\
& \sigma_{z}=C r-E p-D q
\end{aligned}
$$

les équations du mouvement donnent alors d'après les valeurs $0,0, r_{0}$ de $p, q, r$

$$
\begin{aligned}
D r_{0}^{2} & =L, \\
-E r_{0}^{2} & =M, \\
\mathrm{O} & =N .
\end{aligned}
$$

Si $G z$ est un axe principal d'inertie pour $G, L, M, N$ sont nuls et l'action des forces extérieures se ramène à une résultante unique passant par $G$; e'est ce qui arriverait si la masse était sphérique et homogène ou formée de couches concentriques homogènes.

A cause de la variété des mouvements et la nature des masses extérieures, $L, M, N$ ne satisfont pas, quel que soit $t$, aux conditions (2o) et le mouvement d'ensemble est impossible. 